\title{
Dirofilariose canina (Dirofilaria immitis): microscopia óptica e eletrônica de transmissão em lesões da artéria pulmonar de casos naturais
}

\section{Canine dirofilariasis (Dirofilaria immitis): lesions from pulmonary arteries by light microscopy and transmission eletron microscopy, in natural cases}

\author{
Carla Eponina de Carvalho Pinto, ${ }^{*}$ Jefferson Andrade dos Santos, ${ }^{\star \star}$ Eulógio Carlos Queiroz de Carvalho*** \\ Wanderley de Souza ${ }^{\star \star \star \star}$
}

\begin{abstract}
Resumo
As lesões arteriais dos pulmões produzidas pela Dirofilaria immitisforam estudadas por microscopia óptica e microscopia eletrônica de transmissão em 13 cães infectados naturalmente. A microscopia óptica evidenciou projeções papilíferas e/ ou sésseis da íntima, para a luz vascular, algumas vezes acompanhadas por trombos - recentes ou organizados - com restos parasitários. A microscopia eletrônica demonstrou que tais espessamentos estavam associados à presença das células musculares lisas migrantes da média e ao aumento da matriz extracelular. Tais observações, em associação a outros achados, constituem importantes elementos da "Teoria da Proliferação Miointimal", também associada à gênese da ateroesclerose humana.
\end{abstract}

Palavras-chave: Dirofilaria immitis; artéria pulmonar; microscopia óptica; microscopia eletrônica de transmissão.

\section{Introdução}

A Dirofilariose por Dirofilaria immitis é uma doença verminótica, mais comum ao nível do mar de regiões tropicais e subtropicais, zoonótica e transmitida por mosquitos. A sua incidência é alta, principalmente nos cães ( $\mathrm{Ca}$ nis familiaris) e, segundo Rodrigues Silva (1995) em minuciosa revisão bibliográfica, há no mundo registros de 229 casos em humanos, sendo 17 deles no Brasil

A fêmea e o macho adultos da $D$. immitis medem, aproximadamente, 27 e $17 \mathrm{~cm}$ de comprimento, respectivamente. As microfilarias, com aproximadamente $315 \mu$ de comprimento, são liberadas diretamente no sangue do hospedeiro pelas fêmeas. Posteriormente, somente quando sugadas por mosquitos, de espécies váriadas, se desenvolvem de microfilaria para larva infectante, em torno de duas semanas. A infecção ou a reinfecção se dá quando o mosquito pica novamente um hospedeiro definitivo. $A$ forma larvar circulante (microfilaremia) no organismo animal causa no seu trajeto alterações renais e em vasos sangüíneos (Casey e Splitter, 1975; Drazner, 1978; Shirota et al., 1979; Atwell, 1980; Sugimoto et al., 1985; Grauer et al., 1989;). Os adultos parasitam o ventrículo direito e, freqüentemente, o tronco principal da artéria pulmonar de várias espécies de animais domésticos e silvestres, determinando alteração da parede (endarterite e/ou endocardite), do fluxo e da constituição do sangue, culminando com trombose e, às vezes, tromboembolismo, o que pode complicar com hipertensão pulmonar e "cor Pulmonale". A obstrução pós-caval pelo parasita pode determinar congestão passiva da grande circulação, notadamente do fígado (fígado cardíaco). No Homem o parasita adulto tem sido relatado nos pulmões sob a forma de nódulos (Beaufils et al. 1991; Vellayan et al. 1989; Baskin e Eberhard, 1982). Esta investigação centrou-se nos achados ópticos e ultraestruturais, provocados pelo filarídeo adulto, nas camadas da artéria pulmonar e ramificações.

\section{Material e métodos}

\section{Microscopia óptica (MO)}

Lesões de artérias pulmonares de 11 cães mortos naturalmente pela infecção por $D$. immitis, foram levantadas para estudo do arquivo do Serviço de Anatomia Patológica Veterinária Jefferson Andrade dos Santos, da Faculdade de Veterinária da Universidade Federal Fluminense/RJ. As

\footnotetext{
* Profa Assistente de Bioterismo do Dep. de Imunobiologia/UFF (gimcecp@vm.uff.br).

*^ Prof. Titular Emérito de Patologia Veterinária do Dep. de Patologia/UFF.

*** Prof. Titular e Chefe do Setor de Patologia Veterinária/UFF.

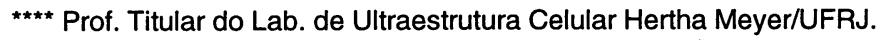


amostras eram de animais necropsiados, de idade, raça e sexo variados e provenientes da região oceânica de Niterói/RJ.

O material, incluído em parafina, foi corado pelo $\mathrm{HxE}$, Tricrômico de Gomori e Weigert-Van Gieson (Behmer et al., 1976).

\section{Microscopia eletrônica de transmissão}

Amostras de lesões vasculo-pulmonares de três animais foram submetidas ao exame ultraestrutural por MET: de dois cães, infectados naturalmente por $D$. immitis, machos, sem raça definida, idades presumidas de 5 anos e apreendidos nas ruas da cidade do Rio de Janeiro; e de um caso de arquivo, eleito como o mais representativo, cujo bloco de parafina foi submetido à técnica de "PopOff" (Schneider et al., 1981).

Os animais com dirofilariose clínica e laboratorial (técnica de Knott) foram sacrificados com overdose de Ketalar e injeção de $5 \mathrm{ml}$ de cloreto de potássio, intravenosos. Na necrópsia foram colhidas amostras de lesões de artéria pulmonar e de seus ramos. Estas mediam $2 \mathrm{~mm} / 2 \mathrm{~mm}$ e foram fixados em solução final de glutaraldeido a $2,5 \%$, mais paraformaldeído a $2 \%$ e tampão cocodilato $0,1 \mathrm{M}, \mathrm{pH}$ 7,3, pós-fixado em solução de uma parte de tetróxido de ósmio $\left(\mathrm{OsO}_{4}\right)$ a $2 \%$ em água, ferrocianeto de potássio $1,6 \%$, cloreto de cálcio $10 \mathrm{mM}$ em uma parte de tampão cocodilato $0,2 \mathrm{M}$; novamente lavados em tampão cocodilato $0.1 \mathrm{M}$, até retirar-se o excesso de ósmio. A seguir os cortes foram desidratados em acetona e incluídos em epon. Dos cortes semifinos $(1 \mu)$ corados com Azul de Toluidina a $1 \%$, foram selecionadas as regiões de interesse, e os ultrafinos $(600 \AA)$, contrastados com acetato de uranila $e$ citrato de chumbo (Souza, 1989).

\section{Resultados}

\section{Microscopia óptica}

Em três animais, a alteração da artéria pulmonar e ramificações consistia em espessamento da íntima, representada por protrusões de caráter séssil e/ou peduncular, para a luz. As projeções pedunculadas, em algumas áreas, mostravam-se reendotelizadas (Fig. 1 e 2) e eram compostas fundamentalmente por material extracelular e células de tecido muscular liso. Observaram-se também, pelo tricrômico de Gomori, fibras musculares lisas (coradas em vermelho), invadindo a íntima e empilhadas perpendicularmente em relação ao endotélio. Estas estavam permeadas por fibras colágenas (Figs. 1, 2 e 3).

Nas lâminas coradas pelo HE massas trombóticas, ora mescladas com hemácias e fibrina, ora constituídas por tecido fibroso hialino com sinais de recanalização, foram observadas nas ramificações da artéria pulmonar (Fig.4). Em dois animais a lesão vascular não se acompanhou de reendotelização e era basicamente constituída por material extracelular. Identificou-se em um animal um filarídeo adulto fêmea livre na luz, com o aparelho reprodutor repleto de microfilárias (Fig. 5).

Dois animais apresentaram, em ramificações da artéria pulmonar, trombos de evolução longa, fibrosos e recanalizados. Em um deles, além da massa trombótica, em outra área as projeções eram de caráter séssil, com fibras colágenas e células musculares lisas. No outro, os trombos fibrinosos eram emaranhados com protuberâncias da íntima, que envolviam restos de filarídeo em degradação ou material lembrando precipitação de minerais.

Em um dos animais a artéria apresentou espessamentos da íntima, variando de sésseis (Fig. 6) a pedúnculopapiliformes (Fig. 7). Estes eram vistos em áreas delimitadas e recanalizadas (Fig. 8). Fibras elásticas invadiam a íntima (Figs. 9 e 10), enquanto as projeções pedúnculopapiliformes eram constituídas, quase que exclusivamente, por fibras musculares (Fig.8). Em algumas áreas, correspondentes à fase inicial do processo lesivo, a íntima

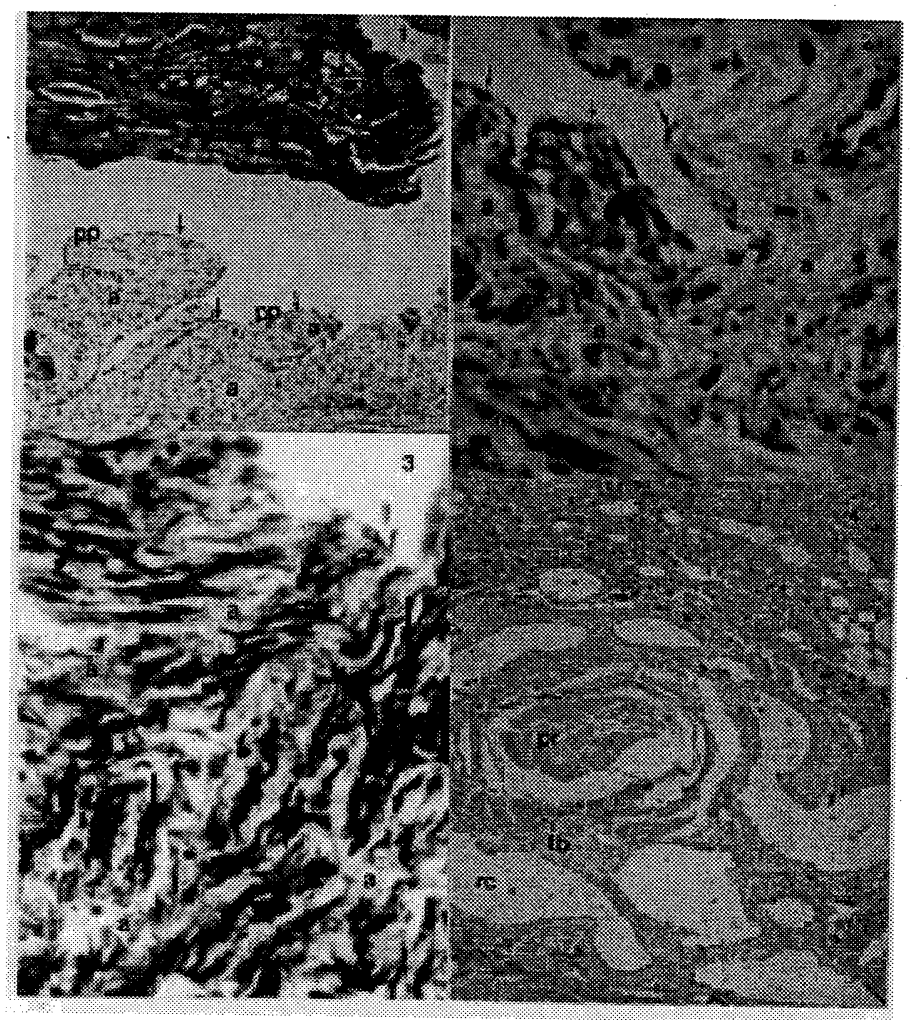

Figuras: 1) Ramificação da artéria pulmonar de cão. Projeções pedúnculo-papilares ( $\mathrm{pp}$ ) reendotelizadas (setas) e compostas basicamente por fibras colágenas (a). Animal $n^{2} 4$, Dirofilariose. "Tricrômico de Gomori". Obj. 10. 0x..; 2) Ramificação da artéria pulmonar de cão. Reendotelização (setas). fibras colágenas (a). Animal no 4, Dirofilariose. "Tricrômico de Gomori". Obj. 40. 0x..; 3) Ramificação da artéria pulmonar de cão. Células musculares lisas, invadindo a intima e empilhadas paralelamente na vertical (setas) e fibras colágenas (a). Animal n⿳3, Dirofilariose. "Tricrômico de Gomor". Obj. 40. Ox..; 4) Ramificação da artéria pulmonar de cão. Trombo (tb) organizado, recanalizado (rc) e com parasito na luz (pr). animal $n^{2} 6$, Dirofilariose. "Hematoxilina-Eosina". Obj. 4.0x.. 


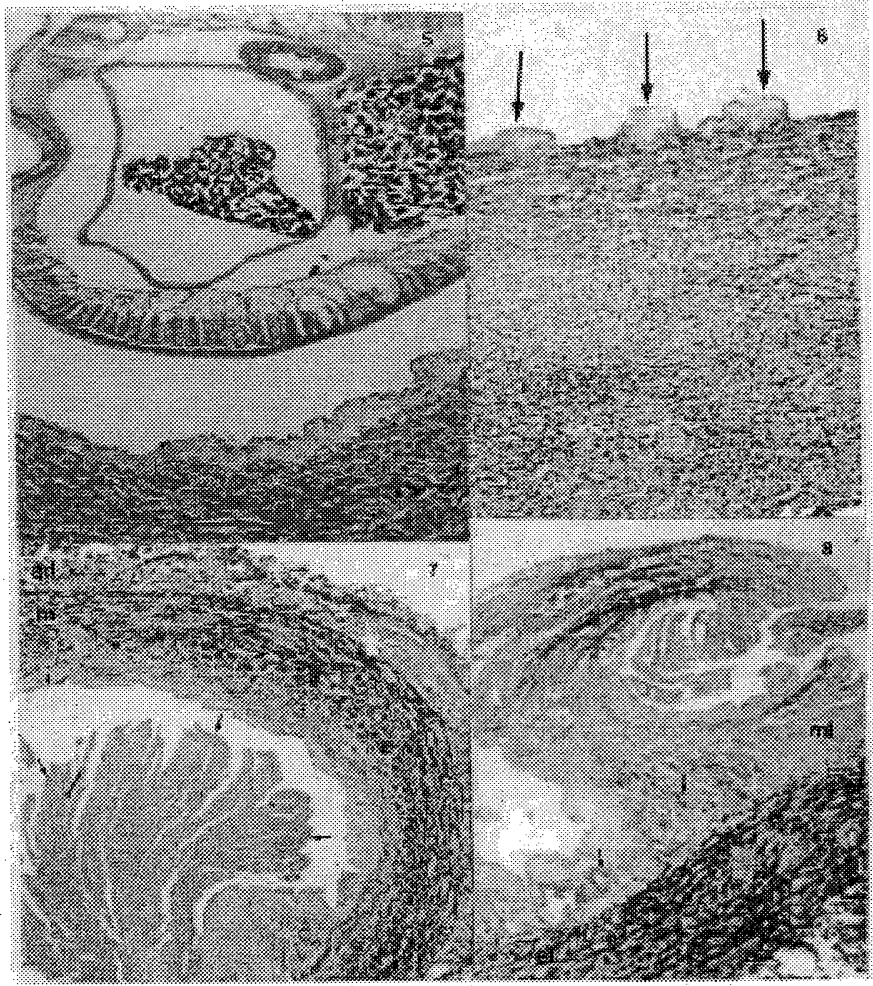

Figuras: 5) Artéria pulmonar de cão. Projeções da íntima espessada. Parasita fêmea, útéro (ut), microfilárias (mf), intestino (int), musculatura (msc), hipoderme (hi), cordão da hipoderme (ch), cutícula (ct). animal no 5, Dirofilariose. "Hematoxilina-Eosina". Obj: 4. Ox. Hematoxilina-Eosina. Obj. 4 0x.. 6) Artéria pulmonar de cão. Projeção séssil da íntima espessada (setas) Animal no 12, Dirofilariose. "Weigert-Van Gieson". Obj. 4.0x. 7) Ramificação da artéria pulomonar de cão. Recanalização da projeção pedúnculo papiliforme da índica (setas). Camadas arteriais: adventícia (ad), média (m) e íntima (i). Animal no 12, Dirofilariose. "Weigert-Van Gieson". Obj. 4.0x.. 8) Ramificação da artéria pulomonar de cão. Recanalização da projeção pedúnculo papilar (setas), fibras musculares lisas, outras estruturas (ml) e elástica (el). Animal no 12, Dirofilariose "Weigert-Van Gieson". Obj. 4.0x..

estava edemaciada (Figs.11 e 12). Notava-se também um espessamento da adventícia com vasa vasorum dilatados.

Um dos casos apresentou restos parasitários em degradação, mineralizados e com cristais de colesterol. Nas ramificações da artéria pulmonar, com a íntima espessa$\mathrm{da}$, as projeções para a luz eram de forma peduncular.

\section{Microscopia eletrônica de transmissão}

Na ultraestrutura de um dos espécimes, identificaram-se microfilárias em capilares pulmonares, constituídas por estruturas internas e cuticulares próprias (Fig. 13).

Nos cortes da artéria pulmonar dos outros dois animais observaram-se células musculares lisas com os núcleos disformes (Fig.14), e citoplasma com prolongamentos (Figs. 14 e 15). Um número expressivo destas células foi observado na camada média migrando em direção à luz do vaso (Fig. 14) e com pseudópodes que estendiam-se através da elástica fenestrada (Fig.15). Migração das cé- lulas musculares lisas para a camada íntima foi observada em um estágio aparentemente recente da lesão. Células endoteliais esparsas cobriam a borda da artéria (Fig. 16). Neste estágio da lesão, o material extracelular era bem escasso e, quando presente, era de natureza colagênica (Figs. 15 e 16).

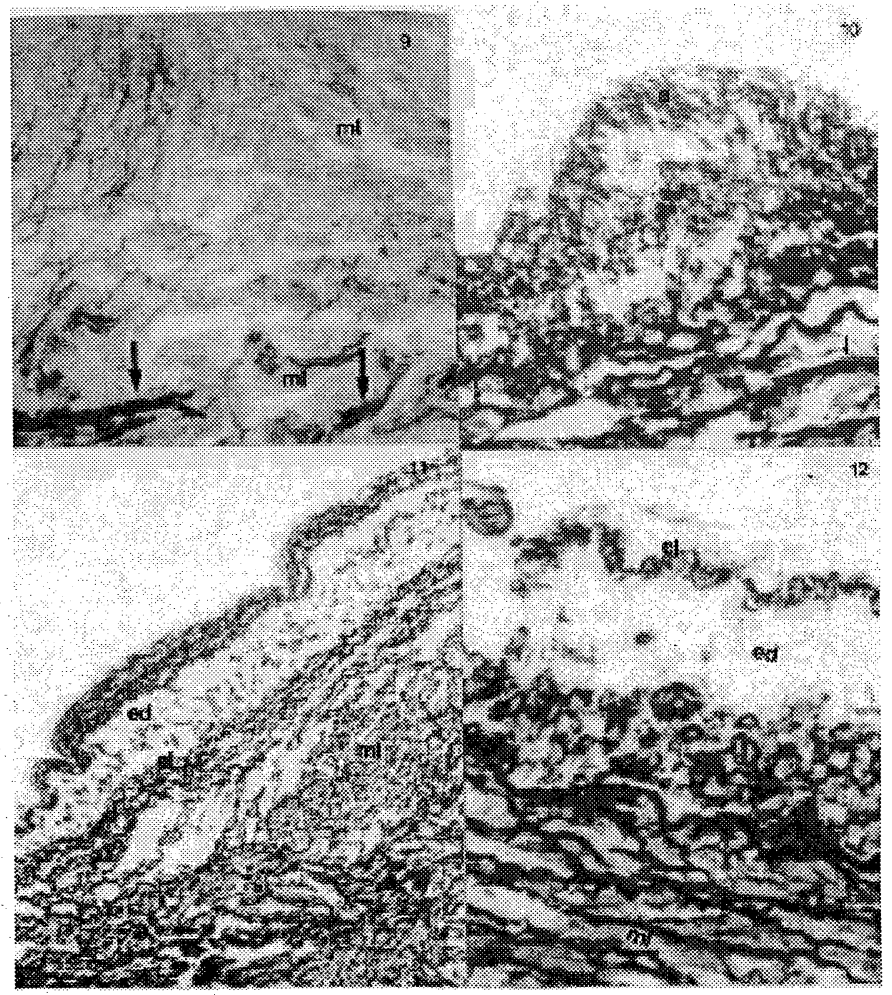

Figuras: 9) Artéria pulmonar de cão. Projeção séssil. Fibras elásticas da íntima (el). Fibras elásticas da camada média (setas). Fibras colágenas (a). Fibras musculares lisas (ml). Animal ne 12, Dirofilariose. "Weigert-Van Gieson". Obj. 40. 0x.. 10) Artéria pulmonar de cão. Projeção séssil. Fibras elásticas na linha espessada (el). Fibras colágenas (a). Fibras elásticas camada média (setas). Fibras musculares lisas (ml). Animal no 12, Dirofilariose. "Weigert-Van Gieson". Obj. 40. 0x..11) Artéria pulmonar de cão. Íntima edemaciada (ed). Fibras musculares lisas (ml). Fibras elásticas (el). Fibras colágenas (a). Animal no 12, Dirofilariose. "Weigert-Van Gieson". Obj. 40. 0x.. 12) Artéria pulmonar de cão. Íntima edemaciada (ed). Fibras elásticas (el). Fibras musculares lisas (ml). Fibras colágenas (a). Animal n 12, Dirofilariose. "WeigertVan Gieson”. Obj. 40. 0x..

O material preparado pela técnica de "Pop-Off" revelou um estágio cronificado da alteração da parede arterial, exibindo um conteúdo nuclear eletrodenso e irregularmente distribuído, sendo também perceptível uma vacuolização citoplasmática. Os vacúolos eram numericamente variáveis e ocasionalmente estavam ocupados por material floculento. Houve tendência destas alterações se apresentarem em células migradas, próximas à região subendotelial, acima ou em pertuitos da elástica interna. O citoplasma destas não tinha prolongamentos e havia sinais de produção de colágeno. 


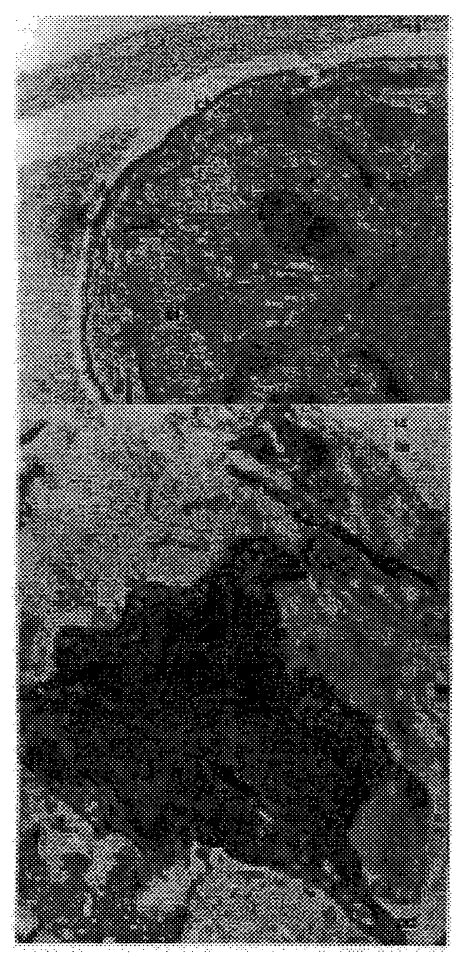

O colágeno constituía-se no material mais abundante de permeio à proliferação intimal, nos diferentes animais estudados ultraestruturalmente. Distribuía-se em toda a extensão da parede vascular, inclusive na íntima, explicando o intenso espessamento visto à microscopia óptica.

Figuras: 13) Capilar pulmonar de cão. Corte longitudinal de uma microfilária. Cutícula (ct). Estruturas internas (ai). Animal $n^{2} 1$, Dirofilariose. 12.000x.. 14) Artéria pulmonar de cão. Célula muscular lisa próxima à luz arterial (la) com núcleo disforme, conteúdo regularmente distribuído $e$ membrana nuclear invaginada (seta). Citoplasma com projeções - pseudópodes (pd). Animal no 1, Dirofila-riose. 12.000x.

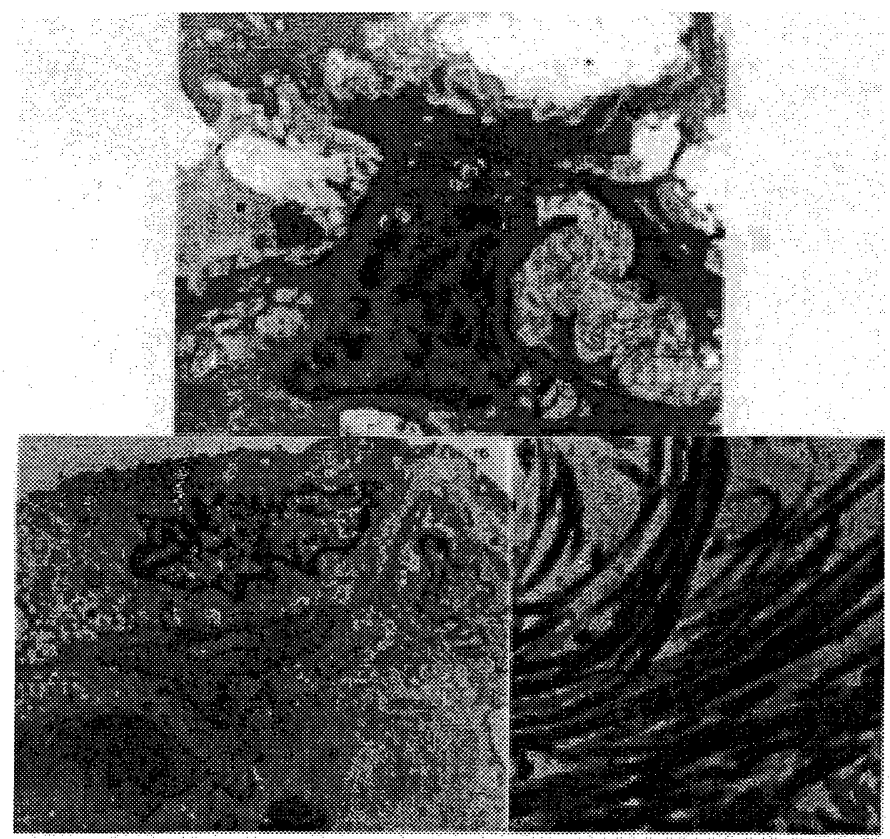

Figuras: 15) Artéria pulmonar de cão. Elástica fenestrada (f). Célula muscular lisa com projeções citoplasmáticas - pseudópodes (pd). Colágeno (a). Animal $n^{2} 1$, Dirofilariose. 7.000x.. 16) Artéria pulmonar de cão. Célula endotelial (ed). Fibras colágenas (a). Animal $n^{\circ} \mathbf{1}$, Dirofilariose. 3000x.. 17) Artéria pulmonar de cão. Fibras colágenas. Técnica de "Pop-Off". Animal no 5, Dirofilariose. 30.000x..

\section{Discussão e conclusões}

A alteração da parede vascular que é iniciada pela ação mecânica e possivelmente tóxica do parasita, desorganiza as células endoteliais e cria assim uma solução de continuidade, expondo colágeno e fibras elásticas (Schaub \& Rawlings, 1980). Este aspecto, não registrado no presente estudo, deveu-se, provavelmente, ao fato do material provir de casos da parasitose espontânea, não tendo sido possível um monitoramento do desenvolvimento cronológico da lesão. Observou-se somente um edema da íntima, enquanto nos trabalhos experimentais são descritas claramente as fases iniciais com a utilização do corante azul de Evans, fundamental na detecção de lesões endoteliais visíveis macroscopicamente a fresco (Rawlings et al., 1981; Keith et al., 1983; Ross, 1986; Barreto et al., 1993; Cotran et al., 1994).

No estágio seguinte, além da exposição dos componentes subendoteliais, as células endoteliais remanescentes, no local, apresentaram fatores ligados a alterações de superfícíe das membranas plasmáticas, que influíram para a adesão de plaquetas. Possivelmente as células aderidas liberaram fatores quimiotáticos que atraíram as células musculares lisas da camada média. A migração mioíntimal e um espessamento da matriz extracelular foram observados na MO através das colorações especiais (tricrômico de Gomori e Weigert-Van Gieson), apresentando uma disposição vertical e paralela dos núcleos das células musculares lisas. A MET, em dois animais, revelou a presença de prolongamentos citoplasmáticos (pseudópodes), projetados além das fenestrações da elástica. Deste modo, as células musculares lisas migraram para a camada íntima. Assim, à medida que a lesão evoluiu, houve maior produção de material extracitoplasmático (colágeno e fibras elásticas), evidenciado na MO (pelas colorações especiais) e MET. O aumento da matriz extracitoplasmática e a migração das células musculares lisas contribui para o espessamento da íntima, com projeções para a luz (Cotran et al., 1994; Ross, 1986; Castleman \& Wong, 1982; Schaub et al., 1983).

Pela MO, nos outros nove animais registrou-se massa trombótica aderida à íntima. Tais achados já tinham sido observados com fibroblastos. Em caso de trombogênese as células musculares lisas têm sido consideradas como precursoras de fibroblastos. No entanto, nas alterações da parede vascular à luz da MET, não foram encontrados os fibroblastos. Acreditamos que o material extracitoplasmático tenha sido sintetizado, pelo menos na sua grande maioria, pelas células musculares lisas (Cotran et al. 1994; Barreto et al. 1993; Munell et al. 1980).

Tais alterações, descritas na reação à injúria do endotélio arterial presente na dirofilariose canina, remontam às bases da "Teoria da Proliferação Miointimal", extensamente investigada com o objetivo de esclarecer os mecanismos de formação do ateroma na aterosclerose humana. Portanto, diante dos achados aqui descritos e pela literatura especializada sobre proliferação miointimal, podemos admitir que as lesões arteriais pela D. immitis constituem um modelo biológico natural para o estudo da aterosclerose humana. E que as células endoteliais e musculares lisas têm uma intensa e imprescindível participação, sendo fundamentais para o desenvolvimento da lesão pela D. immitis na artéria pulmonar e ramificações, como também para aterogênese e trombogênese (Cotran et al., 1994; Barreto et al., 1993; Keith et al., 1983; Schaub et al., 1983). 


\begin{abstract}
Lung vascular lesions of 13 dogs naturally infected with Dirofilaria immitis were studied using light microscopy, transmission electron microscopying and "Pop-Off" technique for paraffin - embedded tissues.

Light microscopy of the pulmonary artery and branches showed papillary and non-papillary intimal proliferations growing towards the lumen of the blood vessel. These intimal proliferations were sometimes associated with recent or organized thrombus containing parasite debris. Under transmission electron microscopy was noticed that such intimal thickenings were due to smooth muscle cells from the medial layer and of extracelular matrix. This observations, in association with other findings, constitute important element of the "Myointimal Froliferations Theory", also relatad to the genesis of human atherosclerosis.
\end{abstract}

Keywords: Dirofilaria immitis; pulmonary arteries; light microscopy; transmission electron microscopy.

\section{Referências bibliográficas}

ATWELL, R. B. Early Stages of Disease of the Peripheral Pulmonary Arteries in Canine Dirofilariasis. Australian Veterinary Journal, v. 56, p.157-159, 1980.

BARRETO, M. N., MONTENEGRO, M. R., BRITO, T., ANDRADE, Z. A. Patologia Processos Gerais. 3. ed. Rio de Janeiro : Atheneu, 1993, 263 p. Cap. 5, p. 85-98.

BASKIN, G. B., EBERHARD, M. L. Dirofilaria immitis Infection in a Rhesus Monkey (Macaca mulata). Lab. Anim-Sci., v. 2, p. 401-402, 1982.

BEAUFILS, J. P., MARTIN, G. J., BERTRAND, F. Dirofilaria immitis Microfilarias in the Urine of a Cat with Urinary Retention. Pratique Medicale \& Chirurgicale de L' Animal de Compagnie, v. 26, p.467-472, 1991.

BEHMER, O. A., TOLOSA, E. M. C., NETO, A. C. F. Manual de Técnicas para Histologia Normal e Patológica, São Paulo, USP, 1976.

CASEY, H. W., SPLITTER, G. A. Membranous Glomerulonephritis in Dogs Infected with Dirofilaria immitis. Veterinary Pathology, v. 2, p. 111-117, 1975.

CASTLEMAN \& WONG. Light and Electron Microscopic in Pulmonary Lesions Associated with Retained Microfilariae in Canine Occult Dirofilariasis. Vet. Pathol., v. 19, p. 355-364, 1982.

COTRAN, R: S., KUMAR, V., ROBBINS, S. L. Robbins Pathologic Basis of Disease ed. Philadelphia: W. B. Saunders Company, 1994, p. Cap. 11, p. 467-516.

DRAZNER, F. H. Renal Amyloidosis and Glomerulonephritis Secondary to Dirofilariasis. Canine Practice, v. 5, p. 66-68, 1978.

GRAUER, G. F.; CULHAN, C. A., DUBIOLZIG, R. R.,LONGHOFER, S. L., GRIEVE, R. B. Experimental Dirofilaria immitis: Associated Gromerulonephritis Induced in Part by "in Situ" Formation of Imune Complexes in the Glomerular Capillary Wall. Journal of_Parasitology, v. 75, p. 585-593, 1989.

KEITH, J. C., SCHAUB, R. G., RAWLINGS, C. Early Arterial Injury - Induced Myointimal Proliferation in Canine Pulmonary Arteries. American Journal Veterinary Research, v. 44, p. $181-186,1983$.
MUNELL, J. F., WELDON, J. S., LEWIS, R. E., THRALL, D. E., McCALL, J. W. Intimal Lesions of the Pulmonary Artery in Dogs with Experimental Dirofilariasis. American Journal Veterinary Research, v. 41, p. 1108- 1112, 1980.

RAWLINGS, C. A., KEITH, J. C., SCHAUB, R. G. Development and Resolution of Pulmonary Disease in Heartworm Infection: Illustrated Review. Journal of the American Animal Hospital Association, v. 17, p. 711-720, 1981.

RODRIGUES SILVA, R.: MOURA, H. \& REY, L. Human pulmonary dirofilariasis: a review. Rev. Med. Trop. SP, v. 37, n. 6, p. 523530, 1995.

ROSS. R. The Pathogenesis of Atherosclerosis - an Update. The New England Journal of Medicine, v. 314, p. 488-500, 1986.

SCHAUB, R. G., RAWLINGS, C. A. Pulmonary Vascular Response During Phases of Canine Heartworm Disease: Scanning Electron Microscopy Study -Am. J. Vet. Res., v. 41, p. 1082-1089, 1980.

SCHAUB, R. G., KEITH, J. C., RAWLINGS. Effect of Acetylsalicylic Acid on Vascular Damage and Myointimal Proliferation in Canine Pulmonary Arteries Subjected to Chronic Injury by Dirofilaria immitis. Am. J. Vet. Res., v. 44, p. 449-454, 1983.

SCHNEIDER, A., BURNS, W., MORRISON, A. "Pop-Off” Technic. The Ultrastructure of Paraffin-embedded Sections. Am. J. Clin. Pathol, v. 4, p. 450-453, 1981.

SHIROTA, A. S., TAKAHASHN, R., FULIWARA, K., HASEGAWA, A. Canine Intersticial Nephritiswith Special Reference to Glomerular Lesions and Filariasis. Jap. J. Vet. Sci., v. 41, p. 119-129, 1979.

SOUZA, W. Manual Sobre Técnicas Básicas em Microscopia Eletrônica. Sociedade Brasileira de Microscopia Eletrônica, Rio de Janeiro: v. I e II., 1989.

SUGIMOTO, J., UNE, Y., SHIROTA, K., IBARAKI, J., NOMURA, Y. Glomerulopathy in Dog Naturally Infected With Dirofilaria immitis. Bulletin of Azabu University Veterinary Medicine, v. 6, p. 105-112, 1985.

VELLAYAN, S., OMAR, B., OTHUMAN, P., JEFFERY, J., ZAHEDI, M., MATHEW, A., KRISHNASAMY, M. The Garden Cat, Felis Temminckii as a New Host for Dirofilaria immitis. Journal Veterinary of Malasya, v. 1, p. 87-89, 1989. 